\title{
TRANSFORMATIONS OF INDEX SET FOR SKOROKHOD INTEGRAL WITH RESPECT TO GAUSSIAN PROCESSES
}

\author{
LESZEK GAWARECKI \\ Kettering University \\ (Formerly GMI Engineering and Management Institute) \\ Department of Science and Mathematics \\ 1700 West Third Avenue, Flint, MI 48504 USA
}

(Received October, 1997; Revised December, 1998)

\begin{abstract}
We consider a Gaussian process $\left\{X_{t}, t \in T\right\}$ with an arbitrary index set $T$ and study consequences of transformations of the index set on the Skorokhod integral and Skorokhod derivative with respect to $X$. The results applied to Skorokhod SDEs of diffusion type provide uniqueness of the solution for the time-reversed equation and, to Ogawa line integral, give an analogue of the fundamental theorem of calculus.
\end{abstract}

Key words: Skorokhod Integral, Anticipative Stochastic Calculus.

AMS subject classifications: $60 \mathrm{H} 05,60 \mathrm{H} 10$.

\section{Introduction}

The purpose of this article is to prove that, in a general case of Gaussian processes and under mild assumptions, transformations of a parameter set do not change the Skorokhod integral and Skorokhod derivative, and to indicate some applications of this fact.

Let $T$ be any set, $C$ a covariance on $T$ and $H(C)=H$ the reproducing kernel Hilbert space (RKHS) on $C$ (note that $H$ may not be separable). With covariance $C$, we associate a Gaussian process $\left\{X_{t}, t \in T\right\}$ defined on $(\Omega, \mathcal{F}, P)$, where $\mathscr{F}=\sigma\left\{X_{t}\right.$, $t \in T\}$. For the details of the constructions above, see [3]. Let $H^{\otimes p}$ be the $p$-fold tensor product of $H$. The $p$-Multiple Wiener Integral (MWI) $I_{p}: H^{\otimes p} \rightarrow L_{2}(\Omega, \mathcal{F}, P$ ) was defined in [6] (see also [5]) as a linear mapping satisfying the following properties. Here $\tilde{f}$ is the symmetrization of $f$.

a) $E I_{p}(f)=0$,

b) $\quad E I_{p}(f) I_{q}(g)=\left\{\begin{array}{cc}0 & \text { if } p \neq q \\ p !(\tilde{f}, \tilde{g})_{H} \otimes p & \text { if } p=q,\end{array} \quad\right.$ for $f \in H^{\otimes p}, g \in H^{\otimes q}$.

c) $\quad I_{p+1}(g h)=I_{p}(g) I_{1}(h)-\sum_{k=1}^{p} I_{p-1}(\underset{k}{g \otimes} h)$, for $g \in H^{\otimes p}, h \in H$. Above, $(g \underset{k}{\otimes} h)\left(t_{1}, \ldots, t_{k-1}, t_{k+1}, \ldots, t_{p}\right)=\left(g\left(t_{1}, \ldots, t_{k-1}, \cdot, t_{k+1}, \ldots, t_{p}\right), h(\cdot)\right)_{H}$. 
We note that $I_{p}(f)=I_{p}(\tilde{f})$ and hence $I_{p}\left(H^{\otimes p}\right)=I_{p}\left(H^{\odot p}\right)$ where $H^{\odot p}$ is the $p$-fold symmetric tensor product.

Let $u, \Omega \rightarrow H$ be a Bochner measurable function with $\|u\|_{H} \in L_{2}(\Omega, \mathscr{F}, P)$. Using Wiener chaos decomposition, $L_{2}(\Omega, \mathscr{F}, P)=\sum_{p=0}^{\infty} \oplus I_{p}\left(H^{\odot p}\right)$, we have a unique representation $u_{t}(\omega)=\sum_{p=0}^{\infty} I_{p}\left(f_{p}(\cdot, t)\right)$, with $f_{p}^{p}(\cdot, *) \in H^{\otimes p+1}$ and $f_{p}(\cdot, t) \in H^{\odot p}$. The Skorokhod derivative and integral of $u$. with respect to Gaussian processes are defined in [6] (for Skorokhod's original definition, see [12]). The Skorokhod derivative $\left\{D_{s} u_{t}, s \in T\right\}$ of $u_{t}$, for a fixed $t$ is an $L_{2}(\Omega, H)$-valued random variable,

$$
D_{s} u_{t}=\sum_{p=1}^{\infty} p I_{p-1}\left(f_{p}\left(t_{1}, \ldots, t_{p-1}, s, t\right)\right) .
$$

The Skorokhod derivative exists iff $E\left\|D . u_{t}\right\|_{H}^{2}=\sum_{p=1}^{\infty} p p !\left\|f_{p}(\cdot, t)\right\|_{H}^{2} \otimes p<\infty$ and $\left\{D_{s} u_{t} \in L_{2}\left(\Omega, H^{\otimes 2}\right), s, t \in T\right\}$, with $H^{\otimes 2}$ identified with the space of HilbertSchmidt operators on $H$, iff $E\left\|D \cdot u_{*}\right\|_{H}^{2} \otimes 2=\sum_{p=1}^{\infty} p p !\left\|f_{p}\right\|_{H}^{2} \otimes(p+1)<\infty$.

The Skorokhod integral of $u$. is an $L_{2}(\Omega)$-valued random variable,

$$
I^{s}(u .)=\sum_{p=0}^{\infty} I_{p+1}\left(\tilde{f}_{p}(\cdot, *)\right) .
$$

We note that $u$. is integrable iff $E I^{s}(u \cdot)^{2}=\sum_{p=0}^{\infty}(p+1) !\left\|\tilde{f}_{p}(\cdot, *)\right\|_{H}^{2} \odot p+1<\infty$.

Example 1: Skorokhod derivative and integral for Brownian motion. In the case of standard Brownian motion, the MWI $I_{p}$ and consequently, the Skorokhod derivate and integral defined above, coincide with the MWI $I_{p}^{i}$, the Malliavin derivative $D^{i}$ and the Skorokhod integral $I^{i}$ defined in [7]. With $V: L_{2}([0,1)] \rightarrow H$ defined by: $V f=\int_{0} f(s) d s$

$$
I_{p}^{i}\left(f_{p}\right)=I_{p}\left(V^{\otimes p} f\right), I^{s}(V(u))=I^{i}(u) \text { and } D_{s}(V(u)(t))=D_{s}^{i} u_{t}
$$

for $f_{p} \in L_{2}\left([0,1]^{p}\right)$ and $u \in L_{2}\left(\Omega, L_{2}([0,1])\right)$. The first two equalities hold in $L_{2}(\Omega)$ and the third holds in $L_{2}(\Omega, H)$ for a fixed $t$.

If $u$ is adapted to the natural (resp. future) filtration of Brownian motion, $\mathcal{F}_{t}=\sigma\left\{B_{s}, s \leq t\right\} \quad\left(\mathcal{F}^{t}=\sigma\left\{B_{1}-B_{s}, t \leq s \leq 1\right\}\right)$, then the Skorokhod and Itô (backward Itỗ) integrals coincide (see [7]).

\section{Skorokhod Integral Under Transformation of a Parameter Set}

For a Gaussian process $\left\{X_{t}, t \in T\right\}$, let $H(X)=\operatorname{cl}\left(\operatorname{span}\left\{X_{t}, t \in T\right\}\right)$, the closure being taken in $L_{2}(\Omega, \mathcal{F}, P)$. With a transformation $R: S \rightarrow T$ we associate a Gaussian process $X^{R}=\left\{X_{R(s)}, s \in S\right\}$ and we call $R$ nondegenerate if it is onto and if $H\left(X^{R}\right)=H(X)$. Our main result on transformations of the Skorokhod derivative and integral is the following:

Theorem 1: Let $\left\{X_{t}\right\}_{t \in T}$ be a Gaussian process and $R: S \rightarrow T$ be a nondegenerate transformation. Denote by $I_{X}^{s}$ and $I_{X}^{s}{ }_{X}^{R}$ the Skorokhod integrals with respect to $X$ and $X^{R}$, respectively. Then:

1) $\quad f_{p} \mapsto f_{p}^{R}=f\left(R\left(s_{1}\right), \ldots, R\left(s_{p}\right)\right)$ is an isometry from $H\left(C_{X}\right)^{\otimes p} \quad$ onto $H\left(C_{X}\right)^{\otimes p}$.

2) If $u \in \mathscr{D}\left(I_{X}^{s}\right)$ then $u^{R}=\left\{u_{R(s)}, s \in S\right\} \in \mathscr{D}\left(I_{X}^{s}\right)$ and $I_{X}^{s}(u)=I_{X}^{s}{ }^{R}\left(u^{R}\right)$. 
Moreover, denote by $D^{X}$ and $D^{X^{R}}$ the Skorokhod derivatives with respect to $X$ and $X^{R}$, respectively.

3) If for $t \in T \quad u_{t} \in \mathfrak{D}\left(D^{X}\right)$, then $u_{s}^{R} \in \mathfrak{D}\left(D^{X^{R}}\right)$ for $s \in R^{-1}\{t\}$ and $D_{s^{\prime}}^{X^{R}}$ $u_{s}^{R}=D_{R\left(s^{\prime}\right)}^{X} u_{R(s)} P$-a.e., for $s, s^{\prime} \in S$. The equality is in $H\left(C_{X^{R}}\right)$, with $s^{\prime} \in S$ as the variable.

Also, $D_{t^{\prime}} u_{t} \in H\left(C_{X}\right)^{\otimes 2},\left(t, t^{\prime} \in T\right)$ implies $D_{s^{\prime}}^{X^{R}} u_{s}^{R} \in H\left(C_{X^{R}}\right)^{\otimes 2}$, $\left(s, s^{\prime} \in S\right)$, and equality of norms $\left.\left\|D_{t^{\prime}} u_{t}\right\|_{L_{2}\left(\Omega, H\left(C_{X}\right)\right.}{ }^{\otimes 2}\right)=$ $\left\|D_{s^{\prime}}^{X} u_{s}^{R}\right\|_{L_{2}\left(\Omega, H\left(C{ }_{X}\right)^{\otimes 2}\right)^{\otimes}}$

4) If $v \in L_{2}\left(\Omega, H\left(C_{X}{ }^{R}\right)\right)$ then $v=u^{R}$ for some $u \in L_{2}\left(\Omega, H\left(C_{X}\right)\right)$ and $\|v\|_{L_{2}}=\|u\|_{L_{2}}$.

Moreover, $v \in \mathscr{D}\left(I_{X}^{s}\right)$ implies $u \in \mathscr{D}\left(I_{X}^{s}\right)$ and $v_{s} \in \mathscr{D}\left(D^{X^{R}}\right)$ implies $u_{R(s)} \in \mathscr{D}\left(D^{X}\right)$ with $D_{s^{\prime}}^{X^{R}} v_{s}=D_{R\left(s^{\prime}\right)}^{X} u_{R(s)}$ for $s, s^{\prime} \in S$.

If $\left.D_{s^{\prime}}^{X^{R} v_{s} \in H(C}{ }_{X^{R}}\right)^{\otimes 2}, \quad\left(s, s^{\prime} \in S\right), \quad$ then $\quad D_{t^{\prime}} u_{t} \in H\left(C_{X}\right)^{\otimes 2}$,

$\left(t, t^{\prime} \in T\right)$, and the $H-S$ norms of those derivatives are equal.

Proof: 1) Let us denote $f^{R}\left(s_{1}, \ldots, s_{n}\right)=f\left(R\left(s_{1}\right), \ldots, R\left(s_{n}\right)\right)$ for $\left(s_{1}, \ldots, s_{n}\right) \in S^{p}$, (thus $\left.f_{p}^{R}\left(s_{1}, \ldots, s_{p}, s\right)=f_{p}\left(R\left(s_{1}\right), \ldots, R\left(s_{p}\right), R(s)\right),\left(s_{1}, \ldots, s_{p}, s\right) \in S^{p+1}\right)$. Let $f(t) \in$ $H\left(C_{X}\right)$, then $f(t)=E\left(X_{t} I_{1}^{X}(f)\right)$, with $I_{1}^{X}(f) \in H(X)$ and, for any $s \in S$,

$$
f^{R}(s)=f(R(s))=E\left(X_{R(s)} I_{1}^{X}(f)\right)=E\left(X_{s}^{R} I_{1}^{X}(f)\right)
$$

$\left(I_{p}^{X}\right.$ or $I_{p}^{X^{R}}$ denotes the $p^{t h}$ order Wiener integral with respect to either $X$ or $\left.X^{R}\right)$. By definition and uniqueness of representation, $f^{R} \in H\left(C_{X}{ }^{R}\right)$ and $I_{1}^{X^{R}}\left(f^{R}\right)$ $=I_{1}^{X}(f)$. Also, if $g \in H\left(C_{X^{R}}\right)$ then, for $s \in S, g(s)=E\left(X_{R(s)} I_{1}^{X^{R}}(g)\right)$. But, $I_{1}^{X^{R}}(g) \in H(X)$, thus $f(t)=E\left(X_{t} I_{1}^{X^{R}}(g)\right)$ defines an element of $H\left(C_{X}\right)$, with $g(s)=f(R(s)), \quad s \in S \quad$ and $\quad\|g\|_{H\left(C_{X}{ }^{R}\right.}=\left\|I_{1}^{X} g\right\|_{L_{2}(\Omega, \mathcal{F}, P)}=\|f\|_{H\left(C_{X}\right)}$, proving (1).

$2)$ - 3) Let us first show that $I_{X}^{p}\left(f_{p}\right)=I_{X}^{p}\left(f_{p}^{R}\right), p=0,1, \ldots$

The above is clear for $p=0$ and $p=1$. Let $f_{p} \in H\left(C_{X}\right)^{\otimes p}, f\left(t_{1}, t_{2}, \ldots, t_{p}\right)=$ $\sum_{\alpha_{1}, \alpha_{2}, \ldots, \alpha_{p}} a_{\alpha_{1}, \alpha_{2}, \ldots, \alpha_{p}} e_{\alpha_{1}}\left(t_{1}\right) e_{\alpha_{2}}\left(t_{2}\right) \ldots e_{\alpha_{p}}\left(t_{p}\right)$, with $\sum_{\alpha_{1}, \alpha_{2}, \ldots, \alpha_{p}}$ $a_{\alpha_{1}, \alpha_{2}, \ldots, \alpha_{p}}^{2}<\infty$ and $\left\{e_{\alpha}, \alpha=1,2, \ldots\right\}$ an ONB in $H\left(C_{X}\right)$. For $f_{p}=e_{\alpha_{1}}\left(t_{1}\right) e_{\alpha_{2}}\left(t_{2}\right) \ldots e_{\alpha_{p}}\left(t_{p}\right)$ we have $\left[\left(f_{p} \underset{k}{\otimes} g_{1}\right)^{X}\right]^{R}\left(s_{1}, \ldots, s_{k-1}, s_{k+1}, \ldots, s_{p}\right)=$ $\left(f_{p}^{R} \underset{k}{\otimes} g_{1}^{R}\right)^{R}\left(s_{1}, \ldots, s_{k-1}, s_{k+1}, \ldots, s_{p}\right)$, where the superscripts $X$ and $X^{R}$ indicate that the operation " $\underset{k}{\otimes}$ " is taken either with respect to the process $X$ or $X^{R}$. Thus, $I_{p}^{X}\left(\left(f_{p} \underset{k}{\otimes} g_{1}\right)^{X}\right)=I_{p}^{X^{R}}\left(\left[\left(f_{p} \underset{k}{\otimes} g_{1}\right)^{X}\right]^{R}\right)=I_{p}^{X^{R}}\left(\left(f_{p}^{R} \underset{k}{\otimes} g_{1}^{R}\right)^{X^{R}}\right)$, which allows us to use the inductive relation $(c)$ for MWI to complete the proof. For $f_{p} \in H\left(C_{X}\right)$ arbitrary, 
we have

$$
\begin{array}{r}
I_{p}^{X}\left(f_{p}\right)={ }_{n_{1}, \ldots, n_{p} \rightarrow \infty} I_{p}^{X}\left(\left(\sum_{\alpha_{1}=1}^{n_{1}} \ldots \sum_{\alpha_{p}=1}^{n_{p}} a_{\alpha_{1}, \ldots, \alpha_{p}} e_{\alpha_{1}} \ldots e_{\alpha_{p}}\right)\right) \\
={ }_{n_{1}, \ldots, n_{p} \rightarrow \infty} I_{p}^{X}\left(\left(\sum_{\alpha_{1}=1}^{n_{1}} \ldots \sum_{\alpha_{p}=1}^{n_{p}} a_{\alpha_{1}, \ldots, \alpha_{p}} e_{\alpha_{1} \ldots e_{\alpha_{p}}^{R}}^{R}\right)\right) \\
=I_{p}^{X}\left(\lim _{n_{1}, \ldots, n_{p} \rightarrow \infty}\left(\sum_{\alpha_{1}=1}^{n_{1}} \ldots \sum_{\alpha_{p}=1}^{n_{p}} a_{\alpha_{1}, \ldots, \alpha_{p}} e_{\alpha_{1} \ldots e^{R}{ }_{\alpha_{p}}}^{R}\right)\right)=I_{p}^{X^{R}}\left(f_{p}^{R}\right) .
\end{array}
$$

Now if $u \in \mathscr{D}\left(I_{X}^{s}\right)$ and $u_{t}=\sum_{p=0}^{\infty} I_{p}\left(f_{p}\left(t_{1}, \ldots, t_{p}, t\right)\right)$ then, for $s \in S$,

$$
u_{R(s)}=\sum_{p=0}^{\infty} I_{p}^{X}\left(f_{p}(\cdot, R(s))\right)=\sum_{p=0}^{\infty} I_{p}^{X^{R}}\left(f_{p}^{R}(\cdot, s)\right)
$$

and 2) and 3) follow.

4) Let $v \in L_{2}\left(\Omega, H\left(C_{X^{R}}\right)\right)$; then for $s \in S$, using 1),

$$
v_{s}=\sum_{p=0}^{\infty} I_{p}^{X^{R}}\left(g_{p}(\cdot, s)\right)=\sum_{p=0}^{\infty} I_{p}^{X^{R}}\left(f_{p}^{R}(\cdot, s)\right)
$$

because for any $g \in H\left(C_{X}\right)^{\otimes(p+1)}$ there exists $f \in H\left(C_{X}\right)^{\otimes(p+1)}$ with $g=f^{R}$. Hence, for $s \in S, v_{s}=\sum_{p=0}^{\infty} I_{p}^{X^{R}}\left(f_{p}^{R}(\cdot, s)\right)=\sum_{p=0}^{\infty} I_{p}^{X}\left(f_{p}(\cdot, R(s))\right)$.

According to 1$), u_{t}=\sum_{p=0}^{\infty} I_{p}^{X}\left(f_{p}(\cdot, t)\right) \in L_{2}\left(\Omega, H\left(C_{X}\right)\right)$ and equality of norms claimed in 4) is satisfied. The last part of assertion 4) follows from 1),2) and 3) since failure to satisfy any stated condition by $u$ implies violation of this condition by $v$.

Example 2: Transformations of parameter set and Skorokhod integral.

1) Brownian motion and time reversal. Let $\left\{u_{t}, t \in[0,1]\right\}$ be an $L_{2}\left(\Omega, L_{2}[0,1]\right)$ valued process adapted to the natural filtration $\left(\mathscr{F}_{t}\right)_{t \in[0,1]}$ of Brownian motion. Note that $\left\{\widetilde{B}_{t}=B_{1}-B_{1-t}, t \in[0,1]\right\}$ is also a Brownian motion and $\left\{\bar{u}_{t}=u_{1-t}\right.$, $t \in[0,1]\}$ is adapted to filtration $\widetilde{\sigma}^{t}=\sigma\left\{\widetilde{B}_{1}-\widetilde{B}_{s}, t \leq s \leq 1\right\}$. Denote $\bar{B}_{t}=B_{1-t}$. We have

$$
\int_{0}^{1} u_{t} d B_{t}=I_{B}^{s}\left(\int_{0}^{\cdot} u_{r} d r\right)=I_{\bar{B}}^{s}\left(\int_{0}^{1-} u r_{r} d r\right)
$$

By the same method as in the proof of Theorem 1 we can show that $I_{\widetilde{B}}^{s}\left(\left(\int_{0} u_{r} d r\right)^{\sim}\right)=I_{B}^{s}\left(\int_{0} u_{r} d r\right)$ with $\left(\int_{0} u_{r} d r\right)^{\sim}=\int_{0}^{1} u_{r} d r-\int_{0}^{1-} \cdot u_{r} d r$. Hence we get

$$
\int_{0}^{1} u_{t} d B_{t}=I_{\widetilde{B}}^{s}\left(\left(\int_{0}^{\cdot} u_{r} d r\right)^{\sim}\right)=I_{\widetilde{B}}^{i}(\bar{u})=\int_{0}^{1} \bar{u}_{t} * d \widetilde{B}_{t}
$$

where "*" denotes the backward Itô integral. We have just obtained the relation 
$I_{B}^{i}(u)=I_{\widetilde{B}}^{i}(\bar{u})$ given in [8]. Note also that $\bar{B}_{t}$ is not a Brownian motion and equation (1) is reversed pathwise in $H$. In the case of Brownian motion, we also have

$$
I_{\bar{B}}^{s}\left(\int_{0}^{1-\cdot} u s_{s} d s\right)=I_{\widetilde{B}}^{s}\left(\left(\int_{0}^{\cdot} u_{s} d s\right)^{\sim}\right) .
$$

Indeed, $\quad I_{\bar{B}}^{s}\left(\int_{0}^{1-} \cdot u_{s} d s\right)=I_{B}^{s}\left(\int_{0} u_{s} d s\right)=I_{B}^{i}(u)=I_{\widetilde{B}}^{i}(\bar{u})=I_{\widetilde{B}}^{s}\left(\int_{0} u_{1-s} d s\right)=$ $I_{\widetilde{B}}^{s}\left(\int_{0}^{1} u_{s} d s-\int_{0}^{1-} \cdot u_{s} d s\right)$.

2. Ogawa Line Integral. We recall the definition of the Ogawa integral $([4,9])$ with respect to a Gaussian process $\left\{X_{t}, t \in[0,1]\right\}$ with the RKHS $H$. Let $u: \Omega \rightarrow H$ be an $H$-valued Bochner measurable function. Then, on a set of $P$-measure one, $u .(\omega)$ takes values in a separable subspace of $H$. Let $\left\{e_{n}, n \in N\right\}$ be an ONB of this subspace. The (universal) Ogawa integral of $u$ is defined as follows:

$$
\delta(u)=\sum_{n=1}^{\infty}\left(u, e_{n}\right)_{H} I_{1}\left(e_{n}\right) \text { (limit in probability) }
$$

if it exists with respect to all ONBs and is independent of the choice of basis.

The relation between Skorokhod and Ogawa integrals is explained in [4].

Let $\gamma: S \rightarrow T$ be a bijective parametrization. Let $Y_{s}=X_{\gamma(s)}$. Then

(i) $\quad C_{X}\left(\gamma\left(s_{1}\right), \gamma\left(s_{2}\right)\right)=C_{Y}\left(s_{1}, s_{2}\right)$;

(ii) $\quad H\left(C_{X}\right)$ and $H\left(C_{Y}\right)$ are isometric under the mapping $f \mapsto f \circ \gamma$;

(iii) $I_{1}^{X}(f)=I_{1}^{Y}(f \circ \gamma)$ for $f \in H\left(C_{X}\right)$.

Thus, $\delta_{X}(u)=\delta_{Y}(v)$ for $v_{s}=u_{\gamma(s)}$, provided either of the integrals exists.

Consider Brownian sheet $\left\{W_{(x, t)},(x, t) \in[0,1]^{2}\right\}$. Assume that $\Gamma \subset[0,1]^{2}$ is a curve parametrized by a function $\gamma:[a, b] \rightarrow \Gamma, 0 \leq a \leq b \leq 1$. We define the Ogawa line integral, $\Gamma-\delta$, over $\Gamma$ with respect to $\left\{W_{(x, t)},(x, t) \in \Gamma\right\}$ using $\Gamma$ as the parameter set. In addition, let $\gamma(s)=\left(\gamma_{1}(s), \gamma_{2}(s)\right)$ with both coordinates nondecreasing and such that the map $\tilde{\gamma}^{-1}\left(\gamma_{1}(r), \gamma_{2}(r)\right)=\gamma_{1}(r) \gamma_{2}(r)$ is bijective from $\Gamma$ to $S=\left[\gamma_{1}(a) \gamma_{2}(a), \gamma_{1}(b) \gamma_{2}(b)\right]$. Then $\tilde{\gamma}: S \rightarrow \Gamma$ is a bijective parametrization and the process $B_{s}=W_{\tilde{\gamma}(s)}$ is a Brownian motion. Hence,

$$
\Gamma-\delta_{W}(u)=\delta_{B}(v)=\int_{S}\left(V^{-1} v\right)(s) \circ d B_{s}
$$

where $v_{s}=u_{\tilde{\gamma}(s)}, V$ is the isometry from Example 1, and the last integral is in the sense of Fisk and Stratonovich and is assumed to exist. In particular, if $u_{(x, t)}=$ $f\left(W_{(x, t)}\right)$ and $f \in C^{2}$, then

$$
\Gamma-\delta_{W}\left(V^{\otimes 2}\left(f^{\prime}(W)\right)\right)=\int_{S} f^{\prime}\left(B_{s}\right) \circ d B_{s}=f\left(W\left(\gamma_{1}(b), \gamma_{2}(b)\right)\right)-f\left(W\left(\gamma_{1}(a), \gamma_{2}(a)\right)\right) .
$$

Thus, in this case, the Ogawa line integral satisfies the fundamental theorem of calculus. We conjecture that a counterpart of Green's formula for the Ogawa integral holds (see [2] for initial exposition and [11] for some recent results). 
Example 3: Skorokhod-type stochastic differential equations. The following class of Skorokhod SDEs was considered by Buckdahn in [1], where, under smoothness assumptions, the author proved existence and uniqueness results

$$
Z_{t}=\eta+\int_{0}^{t} b(Z(s)) d s+I^{i}\left(\sigma(Z(s)) 1_{[0, t]}(s)\right), 0 \leq t \leq 1 .
$$

The initial condition $\eta$ needs to be bounded. However, this restriction vanishes if equation (2) is reversed.

Lemma 1: Let $\left\{u_{s}\right\}_{s \in[0,1]}$ be such that $u_{s} 1_{[0, t]}(s) \in \mathscr{D}\left(I_{B}^{i}\right) \forall t \in[0,1]$. Then for the time reversed process $\bar{u}_{s}=u_{1-s}$, we have $\bar{u}_{s} 1_{[0, t]}(s) \in \mathscr{D}\left(I_{\widetilde{B}}^{i}\right) \forall t \in[0,1]$ and if we denote $X_{t}=I_{B}^{i}\left(1_{[0, t]}(s) u_{s}\right)$, then

$$
X_{1-t}-X_{1}=-I_{\widetilde{B}}^{i}\left(1_{[0, t]}(s) \bar{u}_{s}\right) .
$$

Using time reversal and Lemma 1, Buckdahn's result can be extended to time reversed SDEs with the initial condition being a terminal value of the solution of the original equation.

Theorem 2: Assume that coefficients $b$ and $\sigma$ of a Skorokhod SDE (2) satisfy assumptions for existence and uniqueness of the solution. If $\left\{Z_{t}\right\}_{t \in[0,1]}$ is the solution of Equation (2), then the time reversed process $\bar{Z}_{t}=Z_{1-t}$ is the unique solution in $L_{1}([0,1] \times \Omega)$ of the time reversed equation

$$
X_{t}=\bar{Z}_{0}+\int_{0}^{t}-\bar{b}\left(X_{s}\right) d s+I_{\widetilde{B}}^{i}\left(-1_{[0, t](s)} \bar{\sigma}(X(s))\right)
$$

where $\bar{b}\left(X_{t}\right)=b\left(X_{1-t}\right), \bar{\sigma}\left(X_{t}\right)=\sigma\left(X_{1-t}\right)$, and $\widetilde{B}_{t}=B_{1}-B_{1-t}$.

The above theorem gives a partial answer to a question in [8], Proposition 5.2.

The technique of time reversal has been used in [10] to solve a problem regarding anticipative stochastic models in finance.

\section{Acknowledgements}

The author would like to thank Professor V. Mandrekar for introducing him to anticipative stochastic calculus. The author also thanks the referee for his/her careful reading of the manuscript and providing comments that led to an improved presentation of the paper.

\section{References}

[1] Buckdahn, R., Skorokhod stochastic differential equations of diffusion type, Prob. Theory Related Fields 93 (1992), 297-323.

[2] Cairoli, R. and Walsh, J.B., Stochastic integrals in the plane, Acta Math. 134 (1975), 111-183.

[3] Chatterji, S.D. and Mandrekar, V., Equivalence and singularity of Gaussian 
measures and applications, Prob. Anal. and Related Topics (ed. by A.T. Barucha-Reid), Academic Press, New York 1 (1978), 169-197.

[4] Gawarecki, L. and Mandrekar, V., Itô-Ramer, Skorokhod and Ogawa integrals with respect to Gaussian processes and their inter-relationship, Chaos Expansions, Multiple Wiener-Itô Integrals and Their Applications, CIMAT, Guanajuato, Mexico, July 27-31 (1992), 349-373.

[5] Itô, K., Multiple Wiener integral, J. Math. Soc. Japan 3 (1951), 157-169.

[6] Mandrekar, V. and Zhang, S., Skorokhod integral and differentiation for Gaussian processes, R.R. Bahadur Festschrift, Stat. and Prob. (ed. by J.K. Ghosh, et al.), Wiley-Eastern Limited (1994), 395-410.

[7] Nualart, D. and Zakai, M., Generalized stochastic integrals and the Malliavin calculus, Probab. Theory and Relat. Fields 73 (1986), 255-280.

[8] Ocone, D. and Pardoux, E., A generalized Itô-Ventzell formula. Application to a class of anticipating stochastic differential equations, Ann. Inst. H. Poincaré, Probab. Statist. 25 (1989), 39-71.

[9] Ogawa, S., The stochastic integral of noncausal type as an extension of the symmetric integrals, Japan J. Appl. Math. 2 (1985), 229-240.

[10] Platen, E. and Rebolledo, R., Pricing via anticipative stochastic calculus, $A d v$. in Appl. Probab. 26 (1994), 1006-1021.

[11] Redfern, M., Stochastic integration via white noise and the fundamental theorem of calculus, Stoch. Anal. on Infinite Dimens. Spaces (ed. by H. Kunita, and H.-H. Kuo), Pitman Research Notes in Math Series 310 (1994), 255-263.

[12] Skorokhod, A.V., On a generalization of stochastic integral, Theory of Probab. Appl. 20 (1975), 219-233. 


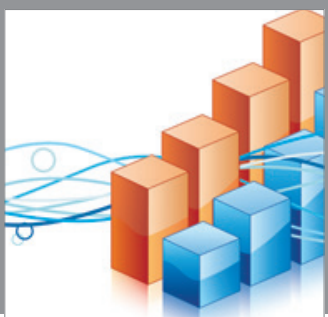

Advances in

Operations Research

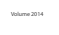

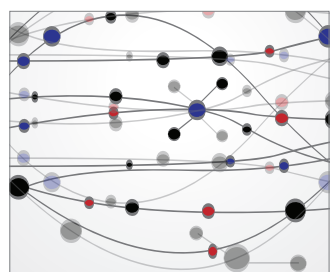

\section{The Scientific} World Journal
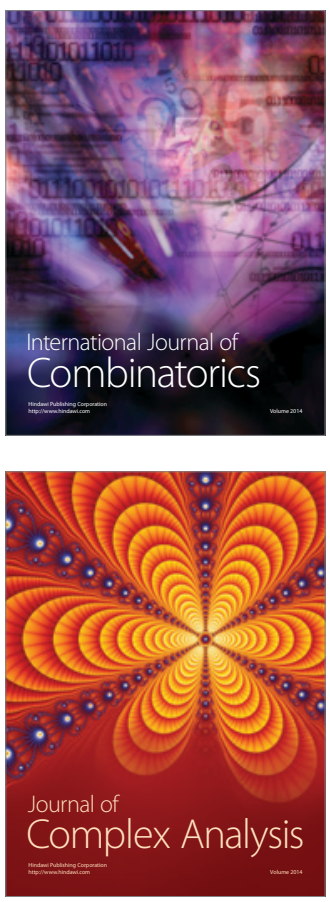

International Journal of

Mathematics and

Mathematical

Sciences
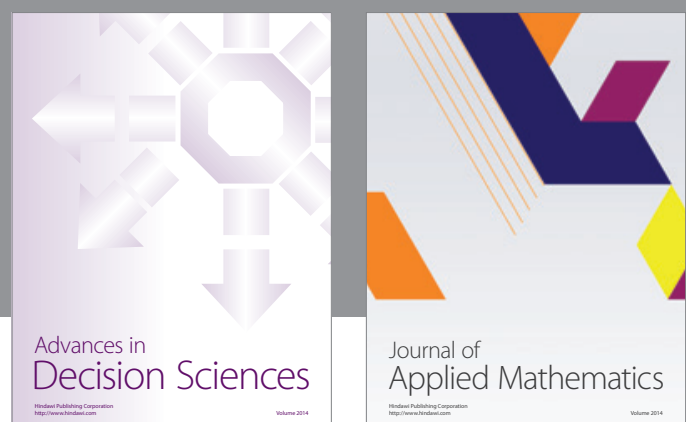

Journal of

Applied Mathematics
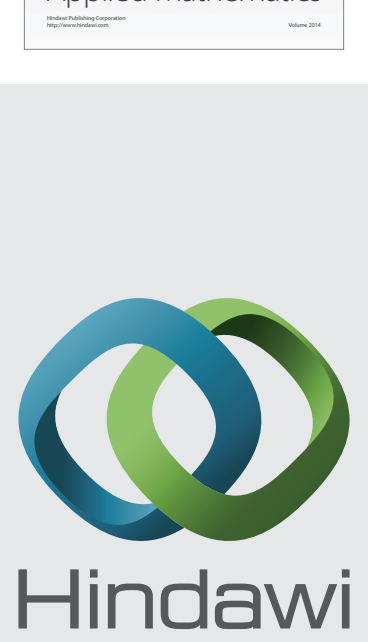

Submit your manuscripts at http://www.hindawi.com
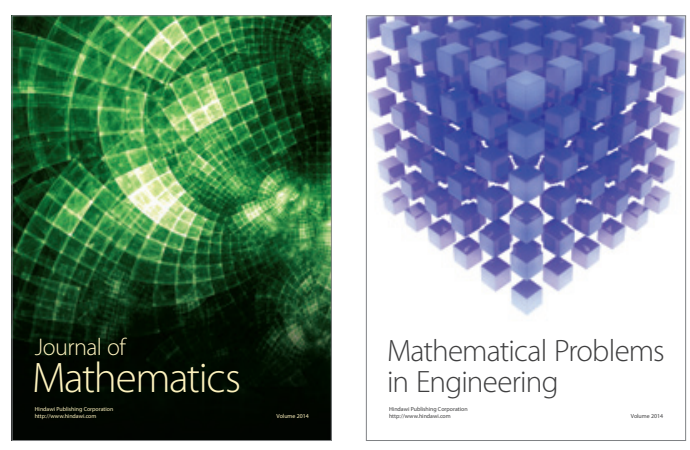

Mathematical Problems in Engineering
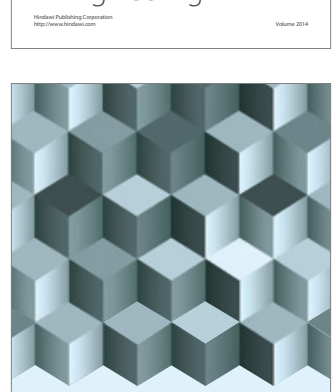

Journal of

Function Spaces
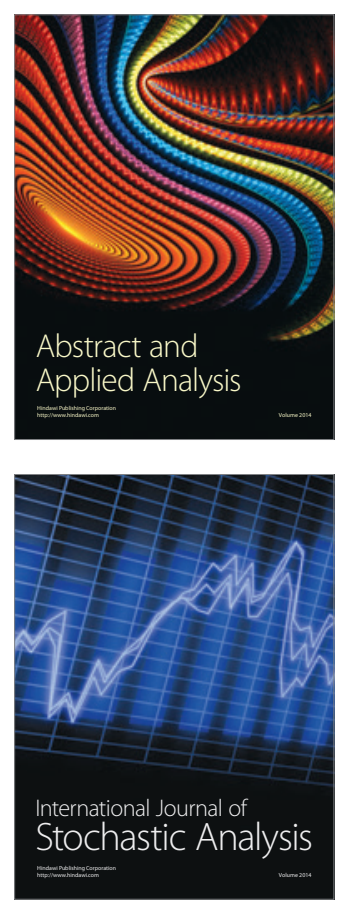

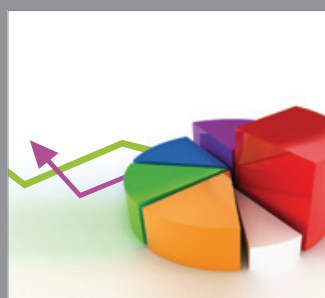

ournal of

Probability and Statistics

Promensencen
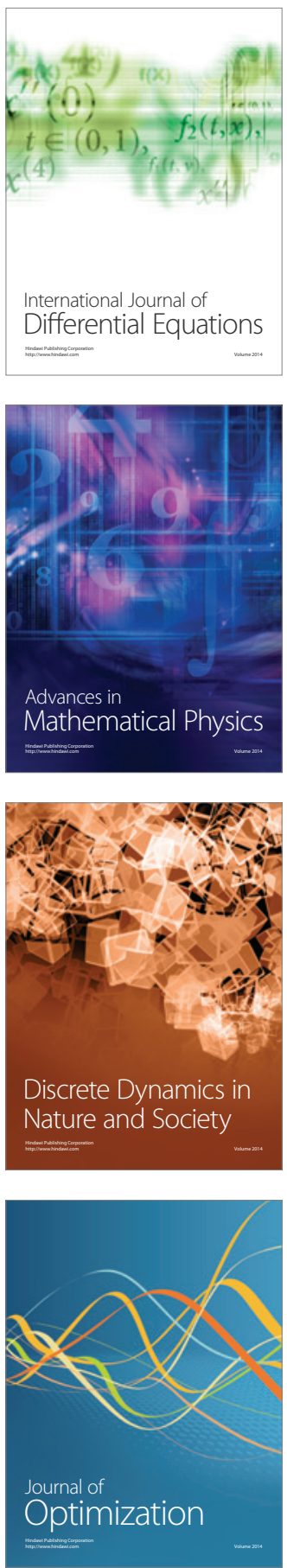